\title{
Evaluation of Pediatric Preseptal Cellulitis Cases Diagnosed with Meningitis by Magnetic Resonance Imaging
}

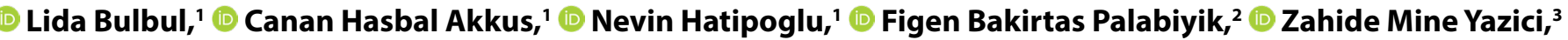 \\ Sadik Sami Hatipoglu' \\ 'Department of Pediatry, University of Health Science Turkey, Bakirkoy Dr. Sadi Konuk Training and Research Hospital, Istanbul, Turkey \\ ${ }^{2}$ Department of Radiology, University of Health Science Turkey, Bakirkoy Dr. Sadi Konuk Training and Research Hospital, Istanbul, Turkey \\ ${ }^{3}$ Department of ENT, University of Health Science Turkey, Bakirkoy Dr. Sadi Konuk Training and Research Hospital, Istanbul, Turkey
}

\begin{abstract}
Rhinosinusitis is a common infection and may rarely cause severe life-threatening orbital and intracranial complications. In this study, two cases with preseptal cellulitis and meningitis as a complication of rhinosinusitis were presented in the light of the literature. A nine years and two months old girl was admitted with complaints as fever, redness and swelling in the left eye. Physical examination revealed erythema and edema in the left lower and upper eyelids, and the eye movements were painless and normal in all directions. Her systemic examination was normal and there was no sign of meningeal irritation. Magnetic resonance imaging revealed ethmoid, frontal and sphenoid sinusitis and left cerebral hemisphere dural meningeal contrast enhancement. The patient was diagnosed with meningitis after lumbar puncture. After 14 days of appropriate antibiotic treatment, the patient recovered and was discharged. An eight years and five months old boy presented with fever, redness and swelling in the left eye was admitted. There were erythema and edema in the left lower and upper eyelid; the eye movements were painless in all directions and were complete. Systemic examination was normal; there was no sign of meningeal irritation. Pansinusitis and preseptal cellulitis findings were detected on computer tomography. The patient's fever persisted under treatment and erythema and edema of the eye became more evident. Orbital MRI was performed considering the complication and contrast enhancement was observed in the left frontal region. The patient was diagnosed with meningitis after lumbar puncture. After 14 days of appropriate antibiotic treatment, the patient recovered and was discharged. Intracranial complication due to preseptal sinusitis is rare but life-threatening. In these cases, we recommend the use of MRI as the radiological imaging method.
\end{abstract}

Keywords: Cellulitis; childhood; meningitis; magnetic resonance imaging.

Please cite this article as "Bulbul L, Hasbal Akkus C, Hatipoglu N, Bakirtas Palabiyik F, Yazici ZM, Hatipoglu SS. Evaluation of Pediatric Preseptal Cellulitis Cases Diagnosed with Meningitis by Magnetic Resonance Imaging. Med Bull Sisli Etfal Hosp 2020;54(4):497-501".

$\mathrm{R}_{\mathrm{c}}^{\mathrm{h}}$ hinosinusitis, which most often develops as a complication of upper respiratory tract infection, is frequently seen both in childhood and adulthood. While only maxillary and ethmoid sinuses are present at birth, sphenoid sinuses develop around the age of five years. Frontal sinuses begin to develop at the age of seven and their develop- ment continues until adolescence. While rhinosinusitis can usually be treated using appropriate treatment methods (oral antibiotics, without antibiotics, nasal decongestants), it has been reported that it may rarely lead to vision loss and even life-threatening orbital and intracranial complications. $^{[1-3]}$

Address for correspondence: Lida Bulbul, MD. Saglik Bilimleri Universitesi, Bakirkoy Dr. Sadi Konuk Egitim ve Arastirma Hastanesi,

Cocuk Klinigi, Istanbul, Turkey

Phone: +90 2124147300 E-mail: doktorlida@yahoo.com

Submitted Date: June 19, 2019 Accepted Date: July 08, 2019 Available Online Date: December 11, 2020

${ }^{\circ}$ Copyright 2020 by The Medical Bulletin of Sisli Etfal Hospital - Available online at www.sislietfaltip.org

OPEN ACCESS This is an open access article under the CC BY-NC license (http://creativecommons.org/licenses/by-nc/4.0/). 
The reason for the frequent occurrence of orbital complications in rhinosinusitis is due to the anatomical neighborhood. In the neighborhood of the orbit, there are sinuses with the frontal sinus at the top, the ethmoidal sinus medially, and the maxillary sinus at the bottom. The paper-thin lamina papyracea, located medially in between with the ethmoidal sinus may cause an infection in the ethmoid sinus to easily spread to the orbit. ${ }^{[4]}$

Orbital complications of rhinosinusitis were first classified by Chandler et al. ${ }^{[5]}$ in the 1970s. This classification continues to be used as a standard in defining orbital complications. Group 1 (preseptal cellulitis) infection affects only the eyelid, Group 2 (orbital cellulitis) is the diffuse edema in orbital structures, Group 3 is the subperiosteal abscess, Group 4 is the abscess development in orbital structures and Group 5 is the infection reaching cavernous sinuses and resulting in cavernous sinus thrombosis. ${ }^{[3,5]}$

Intracranial complications of rhinosinusitis do not have a classification system. It includes epidural or subdural abscess, empyema, meningitis, intracerebral abscess, cavernous and/or sagittal sinus thrombosis involving the central nervous system, particularly the anterior cranial fossa. ${ }^{[3]}$ In the case of underlying immune deficiency, the frequency of intracranial complications is higher, but both orbital and intracranial complications can be seen as a complication of untreated or inadequately treated bacterial rhinosinusitis in immunocompromised patients. ${ }^{[2]}$

In our study, two cases with preseptal cellulitis and meningitis as a complication of rhinosinusitis are presented to draw attention to the intracranial complications of rhinosinusitis.

\section{Case Reports}

Case 1 - A nine years and two months old girl was brought to our emergency clinic with the complaints of fever, redness and swelling in the left eye. Her fever had started two days ago before her admission to the hospital, and the redness and swelling in the eye had started the day before. The patient's history and family history revealed nothing significant, and it was determined that the vaccinations were administered according to the child's chronological age. On physical examination, her general condition was good, she was conscious, and her axillary body temperature was $38.4^{\circ} \mathrm{C}$. There was erythema and edema in the left lower and upper eyelids; her eye movements were painless and normal in all directions. System examinations were normal; there were no signs of meningeal irritation. In the consultation examination performed by ophthalmologists, bilateral vision was normal. In laboratory examinations, blood white blood cell (WBC) count was $12.400 / \mathrm{mm}^{3}$ ( $82 \%$ neutrophils), hemoglobin level was $12 \mathrm{~g} / \mathrm{dL}$, platelet count was 568.000/ $\mathrm{mm}^{3}$, C-reactive protein was $13.9 \mathrm{mg} / \mathrm{dL}$ (normal value $<1$ $\mathrm{mg} / \mathrm{dL}$ ). Blood electrolytes, liver and kidney function tests were normal. With these findings, the patient was hospitalized with a diagnosis of preseptal cellulitis and intravenous (IV) ceftriaxone $(100 \mathrm{mg} / \mathrm{kg} /$ day $)$ treatment was initiated. The patient was consulted with otorhinolaryngology (ENT) department due to increased swelling in the left eye despite treatment. In addition to preseptal cellulitis findings, rhinosinusitis involving ethmoid, frontal and sphenoid sinuses and dural contrast enhancement in the left cerebral hemisphere was detected with contrast-enhanced magnetic resonance (MR) imaging, which was performed to detect possible orbital complications (Fig. 1). Although the patient had no signs of meningeal irritation, lumbar puncture was performed on the second day of hospitalization, with the consent of the parents, to confirm or exclude possible meningitis diagnosis and to determine the duration and choice of treatment. Cerebrospinal fluid (CSF) was clear, with normal pressure, and its protein level was 21 $\mathrm{mg} / \mathrm{dl}$ and glucose level was $63 \mathrm{mg} / \mathrm{dl}$ (simultaneous blood glucose: $110 \mathrm{mg} / \mathrm{dl}$ ). Microscopic examination revealed 18 leukocytes $/ \mathrm{mm}^{3}$ (16 neutrophils). The CSF culture was sent to the laboratory and vancomycin $(60 \mathrm{mg} / \mathrm{kg} /$ day) was added to the ceftriaxone treatment. The patient's fever was reduced on the $3^{\text {rd }}$ day of the treatment. Erythema and edema findings in the eye regressed within days. No significant feature was found in control laboratory examinations. CSF culture was negative. The patient's treatment was completed in 14 days and was discharged with full recovery. The patient's consent was obtained for this study.

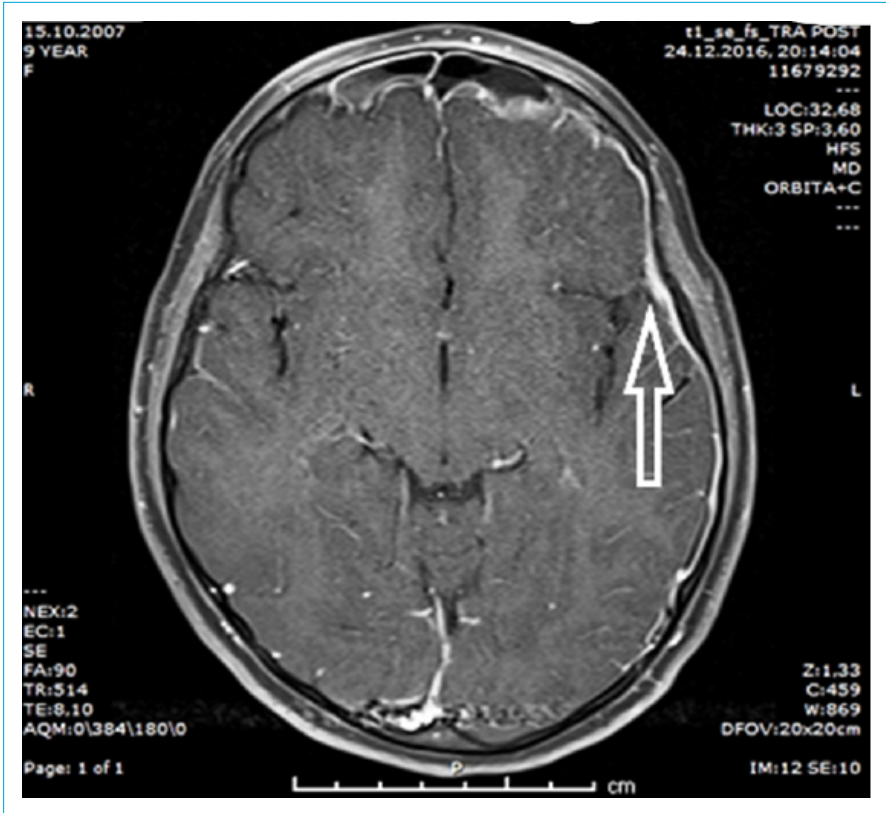

Figure 1. Post-contrast involvement in the left cerebral hemisphere frontoparietal area. 
Case 2 - An eight years and five months old boy was brought with complaints of fever and redness and swelling in the left eye that started the day before. The patient's history and family history revealed nothing significant, and his vaccinations were administered according to the child's chronological age. On physical examination, his general condition was good, he was conscious, and his axillary body temperature was $38.2^{\circ} \mathrm{C}$. He had erythema and edema in the left lower and upper eyelids, and eye movements were painless and normal in all directions. System examinations were normal, and there were no signs of meningeal irritation. In the consultation examination performed by ophthalmology, the anterior segment and fundus were normal, the light reflex was normal, and the vision was 1.0 in the right eye and 0.8 in the left eye. In laboratory examinations, blood WBC count was $19.200 / \mathrm{mm}^{3}$ ( $86 \%$ neutrophils), hemoglobin level was $13.3 \mathrm{~g} / \mathrm{dL}$, platelet count was $290.000 / \mathrm{mm}^{3}$, C-reactive protein was $23.6 \mathrm{mg} / \mathrm{dL}$, and blood electrolytes, liver and kidney function tests were normal. The patient was consulted with the otorhinolaryngology (ENT) department, and computed tomography of the paranasal sinuses was performed to detect the presence of possible orbital complications. Panrhinosinusitis and preseptal cellulitis findings were detected on $\mathrm{CT}$, and orbital abscess was not observed. IV ceftriaxone $(100 \mathrm{mg} / \mathrm{kg} /$ day) treatment was initiated after hospitalization with the diagnosis of preseptal cellulitis. Contrast-enhanced orbital MRI was performed on the patient, whose fever continued on the second day of his admission and whose erythema and edema in the eye increased significantly. Post-contrast examinations showed dural thickening and contrast enhancement in the left frontal region. In the examination of the patient who was receiving antibiotic treatment, there was no change in consciousness and signs of meningeal irritation; however, lumbar puncture was performed on the second day of the treatment to confirm or exclude the possible diagnosis of meningitis and determine the duration and choice of treatment. Cerebrospinal fluid (CSF) was clear, with normal pressure, and its protein level was 20 $\mathrm{mg} / \mathrm{dl}$ and glucose level was $43 \mathrm{mg} / \mathrm{dl}$ (simultaneous blood glucose: $100 \mathrm{mg} / \mathrm{dl}$ ). Microscopic examination revealed 64 leukocytes $/ \mathrm{mm}^{3}$ (60\% neutrophils). The CSF culture was sent to the laboratory and vancomycin $(60 \mathrm{mg} / \mathrm{kg} /$ day) was added to the ceftriaxone treatment. The patient's fever was reduced on the $3^{\text {rd }}$ day of the treatment. Erythema and edema findings in the eye regressed within days. No significant feature was found in control laboratory examinations. The patient's treatment was completed in 14 days and was discharged with full recovery. Vision was normal on control vision examination. The patient's consent was obtained for this study.

\section{Discussion}

While orbital complications are relatively more common in childhood as a complication of rhinosinusitis due to its anatomical neighborhood, the incidence of intracranial complications secondary to rhinosinusitis is extremely rare.

Orbital complications developing in acute childhood rhinosinusitis are reported to be orbital cellulitis, orbital subperiosteal abscess, edema in orbital muscles, inflammation and decreased vision, while intracranial complications include epidural empyema, subdural empyema, intracerebral abscess, meningitis, Pott's puffy tumor and cavernous sinus thrombosis. ${ }^{[6-10]}$

The orbital septum is a thin, fibrous membrane that is anatomically important in the evaluation of orbital infections and acts as a barrier between the eyelids and the orbit. Infections in front of the septum are classified as preseptal, and infections in the posterior part are classified as orbital infections. Preseptal cellulitis develops with near (rhinosinusitis, infections of the eyelid and appendages, direct inoculation with trauma) or distant spread (upper respiratory tract infection, middle ear infection, bacteremia). In preseptal cellulitis, the vision is normal and no afferent pupillary defect or proptosis is detected. Extraocular movements are complete and painless. ${ }^{[4]}$ Both of our cases were brought to our hospital with clinical findings of fever and preseptal cellulitis. Preseptal cellulitis developed due to rhinosinusitis in both patients. In Case 2, the vision in the eye with cellulitis decreased (0.8) at the first examination, but there was no sign of orbital cellulitis in MRI in both cases.

Although there were no signs of confusion, headache, vomiting, or meningeal irritation that would suggest a central nervous system infection clinically in both of our cases, the diagnosis of meningitis was confirmed by lumbar puncture performed upon detection of radiological dural contrast enhancement in MRI. The reason why no growth was detected in CSF culture was might be related to the intravenous antibiotic use of both cases before lumbar puncture. In both patients, meningitis was determined to be caused by the intracranial spread of rhinosinusitis. Case 1 had rhinosinusitis involving ethmoid, frontal and sphenoid sinuses, and Case 2 had panrinosinusitis. In Goytia et al.'s retrospective study of 118 children with complicated sinusitis, intracranial spread was found in 33 patients and orbital spread was found in 85 patients. ${ }^{[6]}$ It was reported that 14 patients with intracranal spread also had orbital spread. Their findings showed that the intracranial spread pattern was as dural thickening in 20 patients, an epidural abscess in 15 patients, as subdural empyema in 15 patients, as frontal bone osteomyelitis in nine patients, as brain abscess in four patients, and as sinus thrombosis in one patient. ${ }^{[6]}$ 
Schlemmer et al. in their retrospective study, conducted with 220 patients, including 138 patients with only orbital complications and 82 patients with intracranial complications with or without orbital findings that were treated for complicated sinusitis between 2006-2009, stated that 65\% of patients with intracranial spread also had orbital complications of sinusitis, most commonly preseptal cellulitis, which was detected as $45 \% \cdot{ }^{[7]}$ Distribution of intracranial complications were reported as follows: $72 \%$ subdural collection, $11 \%$ extradural collection, $6 \%$ subdural+extradural combined collection, $6 \%$ edema/cerebritis and $5 \%$ intracerebral abscess. In this study, they stated that there was a high mortality rate of $20.7 \%$ in patients with intracranial complications, complicated sinusitis was still very common in emerging countries, and that young adolescent males were at the highest risk. ${ }^{[7]}$

Patel et al. evaluated the data of 180 patients in 16 studies in a meta-analysis of intracranial complications of rhinosinusitis in childhood and reported that the most common intracranial complications were subdural empyema (49\%), epidural abscess (36\%), cerebral abscess (21\%) and meningitis (10\%). ${ }^{[8]}$ Padia et al. examined the complications of acute rhinosinusitis in pediatric patients and reported that out of 724 patients diagnosed with acute rhinosinusitis, $29.7 \%$ of 64 patients hospitalized for complications were hospitalized due to intracranial complications (epidural/ subdural abscess, cavernous sinus thrombosis). ${ }^{[9]}$ In another retrospective study conducted with 104 pediatric patients hospitalized with the complication of acute rhinosinusitis, the findings showed that the rate of hospitalization of the male gender and hospitalization in November and March were significantly higher. ${ }^{[10]}$ In the same study, it was reported that the age distribution of patients with only orbital complications was small, compared to those with intracranial complications (mean: 6.5 years/12.3 years). ${ }^{[10]}$ The ages of our two patients confirmed the idea of a lower rate of orbital involvement at an advanced age. In both of our cases, the complication accompanying intracranial involvement was preseptal cellulitis. Both cases were treated successfully with IV antibiotherapy and recovered without any sequelae.

As a radiological examination, paranasal sinus CT was performed at the beginning of the treatment in Case 2, signs of panrhinosinusitis and preseptal cellulitis were detected, but a contrast-enhanced MRI examination was performed upon clinical deterioration under treatment. Although there were no clinical signs of meningitis in both cases, the presence of contrast enhancement on MRI led us to do a further examination and diagnose meningitis. Advanced imaging is also required in patients who do not improve with intravenous antibiotic therapy and whose ophthal- mological examination cannot be performed effectively due to their young age. As radiological imaging, CT is the most widely accepted examination in the diagnosis of rhinosinusitis and shows the presence of complications such as orbital abscess and subperiosteal abscess. ${ }^{[11,12]}$ Patients with signs of orbital infection, such as ophthalmoplegia, proptosis and decreased vision, should be CT scanned to detect an abscess that may require urgent drainage. ${ }^{[4]}$ Magnetic resonance (MR) imaging is the preferred method for detecting intracranial complications, together with MR venography when cavernous sinus thrombosis is considered. ${ }^{[4,11,12]} \mathrm{MRI}$ is more sensitive in evaluating orbital soft tissue complications and intracranial complications of rhinosinusitis. ${ }^{[11-14]}$ This supported the knowledge that MRI is superior in detecting intracranial complications in both of our cases, which helps in the early diagnosis of meningitis without clinical findings.

In conclusion, rhinosinusitis, which is common in childhood, maintains its importance due to its complications. In emerging countries, especially intracranial complications may cause severe mortality. Therefore, rhinosinusitis treatment should be performed effectively with the right antibiotic choices, and patients should be followed up closely, keeping complications in mind. We recommend that patients with orbital complications should be evaluated concerning intracranial complications that may accompany, and MRI should be preferred as a radiological imaging method in cases where intracranial involvement is suspected.

\section{Disclosures}

Informed Consent: Written informed consent was obtained from the patient for the publication of the case report and the accompanying images.

Peer-review: Externally peer-reviewed.

Conflict of Interest: None declared.

Authorship Contributions: Concept - L.B.; Design - L.B.; Supervision - C.H.A.; Materials - N.H., C.H.A.; Data collection \&/or processing - F.B.P., Z.M.Y.; Analysis and/or interpretation - S.S.H., F.B.P.; Literature search - N.H., Z.M.Y.; Writing - L.B., N.H.; Critical review - S.S.H., Z.M.Y.

\section{References}

1. Kronman MP, Crowell CS, Vora SB. Sinusitis. In: Marcdante KJ, Kliegman RM editors. Nelson Essentials of Pediatrics. 8th ed. Philedelphia: Elsevier; 2019. p. 394-5.

2. Hoxworth JM, Glastonbury CM. Orbital and intracranial complications of acute sinusitis. Neuroimaging Clin N Am 2010;20:511-26.

3. Benninger MS, Stokken JK. Acute Rhinosinusitis: Pathogenesis, Treatment, and Complications. In: Flint P, Haughey B, Lund V, Niparko J, Robbins K, Thomas JR, editors. Cummings Otolaryngol- 
ogy. 6th ed. Saunders Elsevier; 2015. p. 724-30.

4. Durand ML. Periocular Infections. In: Bennett JE, Dolin R, Blaser MJ, editors. Principles and Practice of Infectious Diseases. 8th ed. Sounders Elsevier; 2015. p. 1432-8. [CrossRef]

5. Chandler JR, Langenbrunner DJ, Stevens ER. The pathogenesis of orbital complications in acute sinusitis. Laryngoscope 1970;80:1414-28. [CrossRef]

6. Goytia VK, Giannoni CM, Edwards MS. Intraorbital and intracranial extension of sinusitis: comparative morbidity. J Pediatr 2011;158:486-91. [CrossRef]

7. Schlemmer KD, Naidoo SK. Complicated sinusitis in a developing country, a retrospective review. Int J Pediatr Otorhinolaryngol 2013;77:1174-8. [CrossRef]

8. Patel NA, Garber D, Hu S, Kamat A. Systematic review and case report: Intracranial complications of pediatric sinusitis. Int J Pediatr Otorhinolaryngol 2016;86:200-12. [CrossRef]

9. Padia R, Thomas A, Alt J, Gale C, Meier JD. Hospital cost of pediat- ric patients with complicated acute sinusitis. Int J Pediatr Otorhinolaryngol 2016;80:17-20. [CrossRef]

10. Oxford LE, McClay J. Complications of acute sinusitis in children. Otolaryngol Head Neck Surg 2005;133:32-7. [CrossRef]

11. Younis RT, Anand VK, Davidson B. The role of computed tomography and magnetic resonance imaging in patients with sinusitis with complications. Laryngoscope 2002;112:224-9. [CrossRef]

12. Dym RJ, Masri D, Shifteh K. Imaging of the paranasal sinuses. Oral Maxillofac Surg Clin North Am 2012;24:175-89. [CrossRef]

13. Velayudhan V, Chaudhry ZA, Smoker WRK, Shinder R, Reede DL. Imaging of Intracranial and Orbital Complications of Sinusitis and Atypical Sinus Infection: What the Radiologist Needs to Know. Curr Probl Diagn Radiol 2017;46:441-51. [CrossRef]

14. Germiller JA, Monin DL, Sparano AM, Tom LW. Intracranial complications of sinusitis in children and adolescents and their outcomes. Arch Otolaryngol Head Neck Surg 2006;132:969-76. 